\title{
La formación-acción en equipos de trabajo Un estudio de caso con estudiantes de traducción e interpretación en la Universidad de Valladolid
}

\author{
Susana Gómez Martínez \\ Universidad de Valladolid
}

\begin{abstract}
Resumen: El objetivo de este artìculo es describir una enriquecedora experiencia de aprendizaje que se está llevando a cabo en la Universidad de Valladolid con estudiantes de traducciñn e interpretaciñn y expertos en el área participantes de un proyecto de innovaciñn docente de excelencia.

Este proyecto supone una experiencia formativa y colaborativa en la que los estudiantes participantes reciben una formaciñn práctica como complemento a sus estudios (de gran utilidad en su tarea diaria y de cara a su futuro profesional), y los expertos actúan como mentores en una relaciñn horizontal de ayuda, apoyo, confianza y refuerzo positivo caracterìstica del liderazgo transformacional. El objetivo principal es fomentar que los estudiantes aprendan a trabajar en equipo de forma eficaz viéndose a sì mismos como profesionales actuando en un contexto real y significativo en el que se contemplan $\mathrm{y}$ valoran sus ideas, destrezas y fortalezas.
\end{abstract}

Palabras clave: innovaciñn docente, aprendizaje colaborativo, equipos de trabajo, comunidad de aprendizaje, estudiantes de traducciñn e interpretaciñn, liderazgo transformacional, competencias, TICs.

\begin{abstract}
The goal of this paper is to describe a fruitful and enriching learning experience taking place at the University of Valladolid with students of Translation and Interpreting and experts in the field who are participating in an excellence teaching innovation project. This project is aimed at stimulating effective group work and professional development in English through an action-training plan, the use of ICT and following the concept of transformational leadership, an approach which enhances the participants' motivation and performance by inspiring and challenging them to take ownership of their work and learn from each other through cooperative work.
\end{abstract}

Key words: teaching innovation, collaborative learning, learning community, working teams, students of translation and interpreting, transformational leadership, competences, ICTs

Motivaciñn, inspiraciñn, interés, creatividad, colaboraciñn, reto... son algunas de las claves del éxito en educaciñn, pero ¿cñmo podemos fomentar esto entre nuestros estudiantes? 
El enfoque formativo de la experiencia que aquí describimos es la formación - acción, es decir, aprender haciendo y hacer para aprender, de forma que lejos de una formación teórica, la participación en este grupo ofrece a los estudiantes participantes posibilidades de trabajos reales con los que se van a enfrentar los participantes en la actualidad como estudiantes y sobre todo en su futuro profesional: elaboración de traducciones, trabajo en proyectos, reuniones de trabajo, presentaciones en público, presentación de comunicaciones en congresos, creación de materiales, búsqueda de información, trabajo en equipo, coordinación de equipos de trabajo, reparto de tareas, gestión eficaz del tiempo y de las tareas, creación de calendarios y programas de trabajo, uso de las nuevas tecnologías, uso de herramientas informáticas, edición de textos, etc.

De igual modo en esta experiencia de aprendizaje desarrollamos otra serie de competencias fundamentales dentro del EEES: saber trabajar de forma autónoma y en equipo, habituarse a ser responsable y a comprometerse a participar y cooperar en la resolución de problemas y en la toma de decisiones, reflexionar sobre el propio proceso de aprendizaje, aprender a aprender, manejar las herramientas informáticas, desarrollar un método de trabajo organizado y optimizado, desarrollar el espíritu (auto) crítico y creativo, mostrar habilidades de gestión del trabajo y de evaluación del mismo, asumir diferentes roles dentro de un proyecto colaborativo, tomar decisiones, desarrollar la capacidad de comunicarse con otras personas en lenguas extranjeras, desarrollar la capacidad de aplicar los conocimientos y competencias adquiridos en su formación universitaria, etc.

Con esta experiencia pretendemos acercar a los estudiantes participantes a la realidad de los equipos de trabajo y que a su vez experimenten las problemáticas de su futuro ámbito profesional, viéndose a sí mismos como profesionales en foros en los que se contemplan sus apreciaciones razonadas y elaboradas.

Esta formación en competencias, habilidades y destrezas de trabajo en equipo se complementa con el uso del inglés como lengua de trabajo en todo momento (tanto en los trabajos y materiales elaborados como la comunicación oral entre participantes en reuniones, discusiones y tutorías), lo que favorece una práctica extensiva de la lengua en contextos de trabajo reales.

Esta experiencia innovadora interdisciplinar y basada en las metodologías constructivistas (cf. Piaget, 1966, 1970; Glasersfeld 1989, 1995; Kiraly, 2000) ha sido altamente beneficiosa para los estudiantes participantes, tal y como queda latente no sólo en el grado y éxito de consecución de los objetivos que nos marcamos en un principio sino también en la gran implicación y motivación y en las constructivas reflexiones que los estudiantes han realizado sobre lo aprendido y que analizamos también en este artículo.

El objetivo del artículo es describir las claves y objetivos del proyecto y explicar los roles desempeñados por los líderes -los 
expertos- y los participantes -los estudiantes de traducción e interpretación-, tipo de formación que reciben, TICs y herramientas web utilizadas, competencias y destrezas desarrolladas y metodología de trabajo, todo ello ilustrado con reflexiones sobre lo aprendido por parte de los estudiantes participantes.

\section{Objetivos del proyecto}

Tal y como se ilustra en la siguiente figura, nuestro objetivo principal es crear una comunidad de aprendizaje en la que están implicadas otras muchas destrezas, habilidades, competencias y objetivos que favorecen el éxito y el buen funcionamiento de la misma

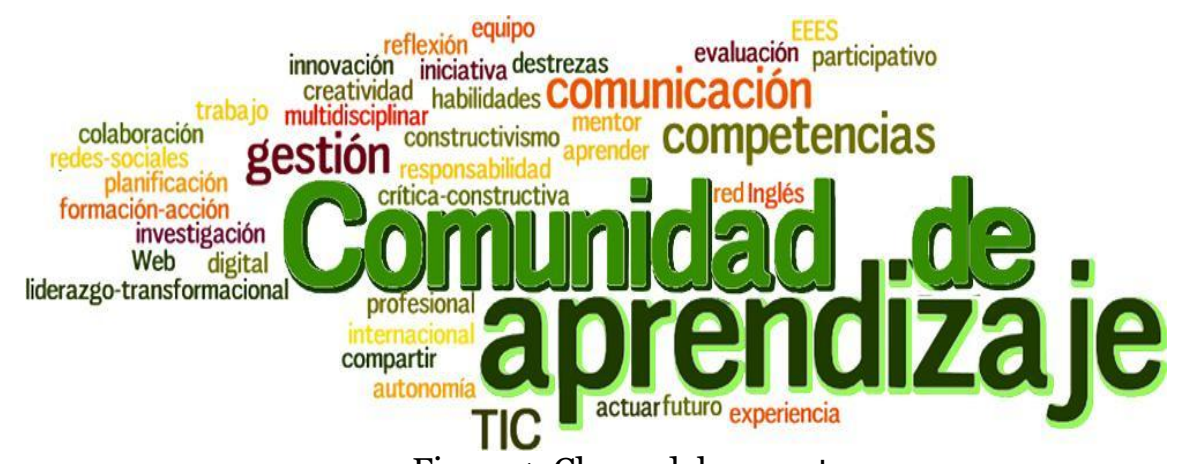

Figura 1. Claves del proyecto siguientes:

Entre los objetivos del proyecto, queremos destacar los

- Establecer un grupo de trabajo entre un profesor -líder y coordinador de esta experiencia- y varios estudiantes de traducción e interpretación

- Mejorar el aprendizaje integral de los estudiantes y su autoestima como futuros profesionales (De Andrés,1999)

- Fomentar que los estudiantes se vean a sí mismos como profesionales actuando en foros en los que se contemplan sus ideas, siguiendo el concepto de liderazgo transformacional (Cf. Hallinger, 2003; Kirby, Paradise, King, 1992).

- Invitar a los participantes a elaborar sus observaciones, argumentos y propuestas, sumergiéndose con ello en la problemática propia del área, lo cual contribuye a su inmersión y desarrollo profesional.

- Lograr que los estudiantes participantes entiendan algunos de los fenómenos y problemas que tienen lugar en su ámbito profesional, así como las implicaciones de los mismos y los posibles mecanismos profesionales para la resolución de estos problemas (cf. Krogerus, Tschäppeler y Guitart, 2001)

- Trabajo del pensamiento crítico y de la crítica constructiva entre los integrantes del equipo, un elemento muy importante para la 
formación y la mejora en el aprendizaje (cf. Bean, 1996; Houston, 2009)

- Tomar conciencia de lo que supone trabajar en grupo en términos de planificación, gestión, comunicación y responsabilidad individual y colectiva, siguiendo las pautas de los expertos en el área (Gavilán y Alario, 2010)

- Impulsar una actitud reflexiva e innovadora hacia el trabajo en grupo desde un enfoque de formación-acción.

- Ofrecer posibilidad de formación a medida de las necesidades del grupo en trabajos reales con los que se van a enfrentar los participantes como estudiantes y sobre todo en su futuro profesional: elaboración de traducciones, trabajo en proyectos, reuniones de trabajo, presentaciones en público, creación de materiales, búsqueda de información, coordinación de equipos de trabajo, reparto de tareas, trabajo en equipo, gestión eficaz del tiempo, eficacia y efectividad en el trabajo, uso de las nuevas tecnologías como parte indiscutible del trabajo diario y la colaboración con otros miembros del grupo, edición y revisión de textos, etc.

- Práctica del inglés como lengua internacional en contextos de trabajo (redacción de correos electrónicos, documentos, etc.). La inmersión en la lengua y el uso de la lengua extranjera en un contexto significativo y real va a ser sin duda un elemento motivador de aprendizaje lingüístico.

- Formación en nuevas tecnologías y en el funcionamiento de entornos virtuales (Moodle) desde el rol del profesor y de herramientas online de gran utilidad para el trabajo en grupo (GoogleDrive, Dropbox, canales YouTube y redes sociales, entre otros (cf. Gisbert, Cabero, Llorente, 2007; Jianhua, y Akahori, 2001; Carrió, M.L. 2007)

Realización de tutorías y reuniones individuales y grupales tanto presenciales como virtuales siguiendo las pautas que los expertos marcan para las reuniones eficaces (Cf. Cantavella y Valls, 2013)

- Fomentar el trabajo autónomo y sobre todo el trabajo colaborativo (cf. Aebli, 1991; Calzadilla, 2002).

- Fomentar el verdadero significado de la palabra equipo: TEAM (together each achieves more), es decir, juntos podemos conseguir algo muy bueno si aunamos nuestras energías y capacidades (Cf. Bress, 2010)

- Reflexionar sobre lo que supone una buena y eficaz gestión del tiempo y de los recursos (cf. Pena, 2009). 


\section{Metodología de trabajo}

La lengua inglesa como vehículo de trabajo es un elemento que tiene una gran importancia en este proyecto. El uso de la misma en contextos auténticos de trabajo ha favorecido enormemente el aprendizaje y la práctica de la misma en un contexto real y significativo.

En cuanto a la metodología de trabajo utilizada, la filosofía del grupo es que los miembros colaboran constructivamente y comparten conocimientos participando en diferentes actividades que eligen voluntariamente en función de su interés y desempeñando un rol que eligen en función de su experiencia y de sus aptitudes. Así pues, cada miembro del equipo elije un rol y apoya a otro compañero en otro rol, disponiendo así de un titular y suplente para poder apoyarse entre sí y aprender entre ellos Los roles y responsabilidades del grupo se detallan a continuación:

- Secretario/a. Es el miembro responsable en organizar y gestionar la información del grupo, garantizar la comunicación entre los miembros del equipo, la entrega de documentación y escribir las actas de las reuniones.

- Técnico audiovisual. Es el miembro experto en comunicación audiovisual y encargado de la toma de fotos y grabación de vídeo

- Diseñador gráfico. Es el experto en diseño gráfico que se encarga del diseño de posters, logos, PPP, y material gráfico vario.

- Asistente técnico para el uso de herramientas informáticas. Es un miembro conocedor de las nuevas tecnologías, hábil en el manejo de los ordenadores, con programas informáticos, edición de vídeo etc.

- Revisor de textos. Es la persona encargada de releer o revisar el material creado por el grupo para evitar que haya erratas.

- Animador/coordinador estudiantil. Es la persona que promueve la colaboración de otros estudiantes de fuera del grupo y les anima a participar en otras actividades.

- Investigador para buscar información. Es el miembro encargado de buscar información en la web, bibliografía, y cualquier tipo de material necesario para el proyecto. 


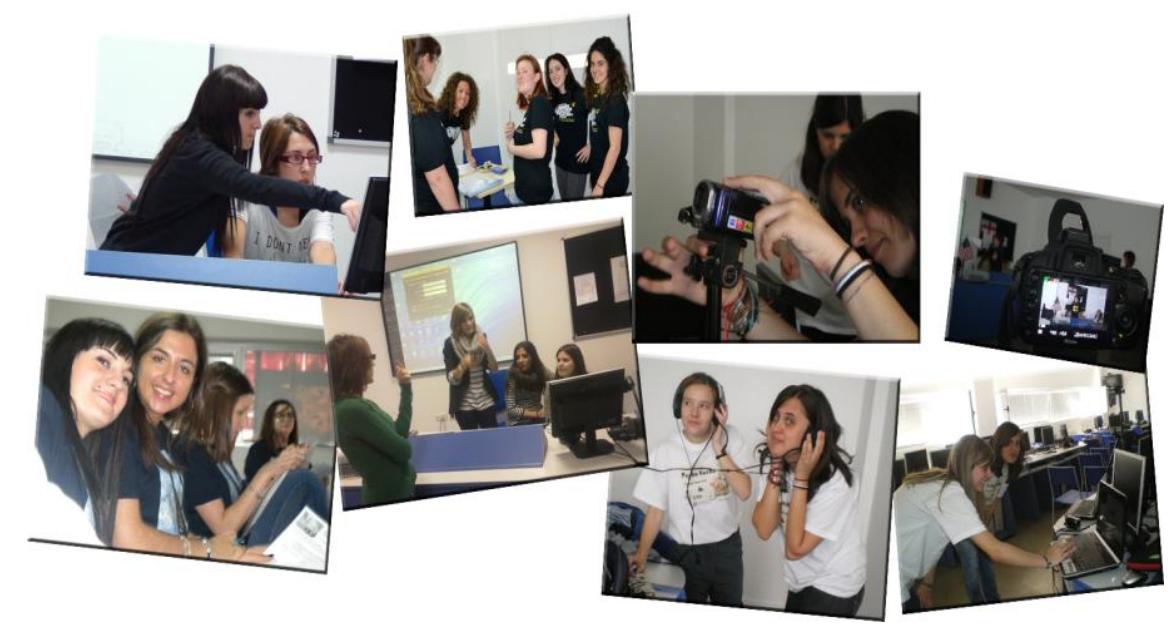

Figura 2. Imágenes de la experiencia de trabajo colaborativo de los estudiantes

\section{Opiniones de los estudiantes participantes}

Consideramos que el nivel de éxito de una propuesta se debe medir con el grado de satisfacción del público meta al que va dirigido. De ahí que consideremos oportuno resaltar alguno de los comentarios más significativos que de su experiencia han realizado los estudiantes participantes y que ilustran muy bien lo aprendido en el mismo.

Teniendo en cuenta que el objetivo principal a la hora de recabar la información era conseguir una información lo más fiable posible, los comentarios son anónimos.

- Supone una oportunidad fantástica para conocer a gente interesante de otros cursos y con otras perspectivas. La gente que he conocido en esta experiencia tiene buenas aspiraciones y la capacidad de trabajo para perseguirlas, trabajar con ellas ha sido una inspiración.

- Gracias a este proyecto he aprendido que el trabajo en grupo no consiste solo en juntarse para realizar una TAREA, sino en cooperar para lograr un OBJETIVO COMÚN, lo cual permite a personas ordinarias conseguir resultados extraordinarios que a nivel individual resultarían inviables. Además he aprendido que pese a que la idea de "trabajo en grupo" implica "colectividad", el compromiso individual de cada miembro del grupo es esencial para el éxito del trabajo. Asimismo, estoy totalmente convencida de que trabajar en este proyecto con otras compañeras me ha enseñado por encima de todo a ser más tolerante, paciente, respetuosa y a tener una mentalidad más abierta a otras ideas y opiniones con las que nunca me habría topado si hubiera trabajado sola. Por último, he aprendido que el trabajo en grupo es muy positivo, ya que siempre tienes a 
personas de tu lado, lo cual te permite minimizar tus puntos débiles y maximizar tus puntos fuertes.

- Viendo los resultados del trabajo, me siento orgullosa. El grupo ha logrado objetivos que a priori parecían inalcanzables. Hemos desarrollado una dinámica de grupo basada en la confianza entre miembros y hemos logrado que el trabajo se llevase a cabo incluso cuando no era posible una reunión presencial. He visto que la opinión de mis compañeras es inestimable y que el aprendizaje trasciende los muros de la universidad. He aprendido de los fallos, aciertos y consejos de mis compañeras y he aprendido de mis propios errores y aciertos. Esta ha sido una experiencia muy completa que abarca muchos campos del conocimiento y que prepara a sus miembros para el mundo laboral de una forma atractiva, amena y activa, especialmente por su cualidad colaborativa.

- He aprendido que el trabajo colaborativo va mucho más allá de los trabajos en grupo que te suelen mandar en el aula. Con este proyecto, he aprendido a valorar las diferentes aportaciones que realizaban mis compañeros y he sabido aportar al grupo aquello que me hacía diferente a los demás. Y es que no siempre por hacer más estás dando más al grupo. Tienes que saber valorar cuándo pedir ayuda y cuándo delegar.

- A raíz de mi participación en esta experiencia puedo afirmar que el aprendizaje colectivo me ha enseñado a trabajar en grupo con mis compañeros, a incluir nuevas ideas y visiones en mi forma de realizar una determinada tarea y a fomentar el conocimiento mediante el trabajo compartido. Y para llevar a cabo dicho trabajo y evaluarlo posteriormente, se ha hecho precisa la supervisión en todo momento de un mentor que nos diese la información y los materiales, además de transmitirnos apoyo y ánimo, que es precisamente el papel que ha desempeñado la coordinadora de este proyecto. No podría haber estado más contenta de haber compartido esta experiencia con mis compañeras de equipo, que han sabido ayudarme en todo momento y confiar en mí para la realización de las diferentes tareas que hemos llevado a cabo de manera conjunta. También he aprendido a saber respetar el trabajo de los demás y a valorar todos los puntos de vista, lo cual es algo muy positivo para el futuro laboral.

- .Esta experiencia me ha ofrecido muchas lecciones sobre el mundo profesional. También he aprendido que el trabajo en equipo es real fuera de la universidad y puede producir unos resultados espectaculares si se fomenta la cooperación

- Cualquier tipo de crítica o comentario repercutirá positivamente en el resultado final 
- (...) al principio me resultó extraño el método de trabajo, pero a la larga te das cuenta de que es lo más productivo y flexible, además de lo que más se adecua a la realidad profesional. Otra cosa que he aprendido en es a hacer frente a las cosas de última hora, algo muy común en el mundo profesional, pero no tanto en el ámbito académico

- La trasmisión del conocimiento y la organización en conjunto ha supuesto para mí trabajar codo con codo, sumar esfuerzos y competencias con el fin de lograr un objetivo común. Este trabajo en equipo ha creado una interdependencia positiva, es decir, pensar en "nosotros" en vez de en el "yo", y una responsabilidad de compromiso individual.

- Emplear el inglés como lengua de trabajo obliga a consultar fuentes como corpus o diccionarios para corregir posibles errores y así se aprende de forma continua y progresiva. De igual modo, la comunicación entre nosotros en inglés a través del correo electrónico ayuda a memorizar muchas estructuras fijas que pueden ser de utilidad en un futuro y en un contexto real. El hecho de que todas las reuniones de grupo sean también en inglés ayuda a la hora de asimilar nuevos conceptos y de facilitar la fluidez en dicha lengua.

- A raíz de formar parte de esta experiencia he descubierto un sinfín de herramientas que facilitan enormemente el trabajo tanto individual como en grupo. En el ámbito académico, si bien es verdad que están cada vez más presentes, no se les da la importancia que merecen, pues son en día a día en el ámbito laboral.

- Antes de formar parte de este grupo no conocías las ventajas de GoogleDrive. Ahora no concibo otro medio de trabajo para estar intercomunicado con los miembros de mi equipo de trabajo, no solo de este grupo, sino también a nivel académico.

- Nuestras reuniones no han podido ser siempre presenciales, por lo que hemos ganado experiencia en videoconferencias y en el manejo del correo y los foros como una herramienta de comunicación rápida y efectiva. La comunicación es una parte clave de esta experiencia.

- Trabajar en ello me ha proporcionado un conocimiento de primera mano que no se aprende en libros o en la universidad.

- Mientras hemos trabajado en el grupo hemos tenido la oportunidad de comparar su funcionamiento con el de los grupos de trabajo de clase y hemos visto unas diferencias notables en la dinámica entre compañeros. Esto nos ha llevado a poner en relieve nuestros puntos fuertes y a plantear la opción de compartir nuestra dinámica de grupo. 
A través de estos comentarios que han dejado los participantes de esta experiencia, consideramos que, no sólo queda latente la filosofía detrás de la misma, sino a su vez el éxito del objetivo principal: crear una comunidad de aprendizaje.

\section{Conclusiones}

La experiencia que hemos presentado en este artículo es muy innovadora por los siguientes motivos: supone un proceso de mejora de carácter integral, gracias a su interdisciplinariedad y al desarrollo de competencias, habilidades y destrezas de tipo trasversal del estudiante, las líneas de trabajo están claramente definidas con metas a corto, medio y largo plazo y garantiza la formación de los estudiantes participantes mediante una formación continua que favorece a su vez la formación permanente.

De igual modo, como aspectos innovadores, nos gustaría destacar los siguientes: fomenta la autonomía en el aprendizaje promoviendo la iniciativa y creatividad de los componentes del grupo; fomenta el trabajo colaborativo a través de una comunidad de aprendizaje; proporciona al profesorado una visión real tanto de los intereses y necesidades de los estudiantes como de sus preferencias a la hora de aprender; fomenta el uso y la práctica del inglés como lengua internacional de trabajo; fomenta un acercamiento a las tareas profesionales en un contexto altamente significativo ya que acerca a los estudiantes participantes a la realidad de los equipos de trabajo y les permite experimentar las problemáticas de su futuro ámbito profesional, viéndose a sí mismos como profesionales en foros en los que se contemplan sus apreciaciones razonadas y elaboradas.

De igual modo, esta experiencia supone además un espacio para los estudiantes participantes de relación, cooperación, apoyo, socialización, comunicación, internacionalización y formación continua,

\section{Bibliografía}

Aebli, H. (1991). Factores de la enseñanza que favorecen el aprendizaje autónomo. Madrid: Narcea.

Bean, J. C. (1996). Engaging ideas: The professor's guide to integrating writing, critical thinking, and active learning in the classroom. San Francisco: Jossey-Bass.

Bress, P. (2010). "Teamwork". English Teaching Professional, 67:48-54

Calzadilla, M.E. (2002). Aprendizaje colaborativo y tecnologías de la información y la comunicación. Revista Iberoamericana de Educación [disponible en línea]: http://www.rieoei.org/deloslectores/322Calzadilla.pdf >. [Fecha de consulta: 27/01/2011].

Cantavella, E. y Valls A. (2013). Reuniones Eficaces: 25 claves para pasar de reunirse a reunirse. Profit Editorial. 
Carrió, M.L. (2007). Ventajas del uso de la tecnología en el aprendizaje colaborativo. Revista Iberoamericana de Educación, 41/4: 1-10.

De Andrés, V. (1999). Self-esteem in the classroom. En J. Arnold (Ed.) Affect in Language Learning (pp) . Cambridge. Cambridge University Press.

Gavilán, P. y Alario, R. (2010). Aprendizaje cooperativo. Una metodología con futuro. Principios y aplicaciones. Madrid: CSS.

Gisbert, M. , Cabero, J. y Llorente, M.C. (2007). El papel del profesor y el estudiante en los entornos tecnológicos de formación. En J. Cabero (Coord.) Tecnología Educativa, (pp. 263-281). Madrid: McGraw Hill.

Glasersfeld, E.V. (1989). Constructivism in education. En T.Husen y N. Postlethwaite(comps.), International encyclopedia of education (11-12). Oxford: Pergamon.

Glasersfeld, E.V. (1995). A constructivist approach to teaching. En L. P. Steffee \& J. Gale (comps.), Constructivism in education (3-16). Hillsdale: Erlbaum.

Hallinger, P. (2003). "Leading Educational Change: reflections on the practice of instructional and transformational leadership". In: Cambridge Journal of Education 33 (3): 329-352.

Houston, H. (2009a). Provoking Thought. Charleston: Anthimeria Press

Jianhua, Z. y Akahori, K. (2001). Web-Based Collaborative Learning Methods and Strategies in Higher Education. Actas del congreso $2^{\text {nd }}$ International Conference on Information Technology Based Higher Education and Training. Kumamoto, Japan, July 4-6, 2001.

Kiraly, D. (2000). A Social Constructivist Approach to Translator Education. Enpowerment from Theory to Practice. Manchester (UK) \& Northhampton MA.: St. Jerome Publishing

Kirby, P.C., Paradise, L.V., King, M.I. (1992). "Extraordinary Leaders in Education: Understanding Transformational Leadership". In: The Journal of Educational Research, 85 (5): 303-311

Krogerus, M., R. Tschäppeler y S. Guitart, (2001) El pequeño libro de las grandes decisiones: 50 modelos para el pensamiento estratégico: Grupo Planeta.

McGarrell, H. y Verbeem, J. (2007). Motivating revision of drafts through formative feedback. ELT Journal, 61(3), 228-236Cebrián

Pena, A. (2009). Gestiona Mejor tu Vida. Claves y hábitos para ser más productivo. Libros Libres.

Piaget, J. (1966). Psychologie der Intelligenz. Zürich: Rascher.

Piaget, J. (1970). Piaget's theory. En P.MUSSEN (comp.), Carmichael's manual of child psychology (703-732).New York:Wiley. 